\title{
Fixed point results for admissible mappings with application to integral equations
}

\author{
Huaping Huang ${ }^{a, *}$, Guantie Deng ${ }^{a}$, Stojan Radenovićb ${ }^{\text {, Zhanmei Chen }}{ }^{a}$ \\ ${ }^{a}$ School of Mathematical Sciences, Beijing Normal University, Laboratory of Mathematics and Complex Systems, Ministry of \\ Education, Beijing, 100875, China. \\ ${ }^{b}$ Faculty of Mechanical Engineering, University of Belgrade, Kraljice Marije 16, 11120, Beograd, Serbia.
}

Communicated by W. Shatanawi

\begin{abstract}
This work is intended as an attempt to improve and simplify some recent fixed point theorems for (weak) $\alpha$-admissible mappings and $(\alpha, \beta)$-admissible mappings from several articles in the framework of $b$-metric spaces. An application in proving the existence of solution for a class of nonlinear integral equations is given. (C)2016 all rights reserved.
\end{abstract}

Keywords: $\alpha$-admissible mapping, fixed point, $(\alpha, \beta)$-admissible mapping, $b$-continuous, integral equation. 2010 MSC: 47H10, 54H25.

\section{Introduction and preliminaries}

Let $(X, d)$ be a complete metric space and $f: X \rightarrow X$ a mapping. If there exists a real number $k \in[0,1)$ such that for all $x, y \in X$, the following inequality holds:

$$
d(f x, f y) \leq k d(x, y)
$$

then $f$ has a unique fixed point in $X$. This famous result is called Banach contraction mapping principle. This principle was found by Polish mathematician Banach in 1922 (see [3]), and it is one of the most important theorems in the field of fixed point theory. Due to its strong applications in proving the existence and uniqueness of solution of integral equations, people have looked more closely at it for many years.

\footnotetext{
${ }^{*}$ Corresponding author

Email addresses: mathhhp@163.com (Huaping Huang), denggt@bnu.edu.cn (Guantie Deng), radens@beotel.net (Stojan Radenović), zhanmei9920106@sina.cn (Zhanmei Chen)
} 
Notice that, the contractive condition 1.1 is satisfied for all $x, y \in X$, which forces the mapping $f$ to be continuous, and so the principle is not applicable on condition that $f$ is discontinuous. By virtue of such drawback, it is sometimes inconvenient to carry out this principle. Recently, several authors attempted to overcome this difficulty, see, e.g., Ran and Reurings [12], Kirk et al. 9], Jachymski [7] and the recent results of Samet et al. [15. The notion of $\alpha$-admissible mapping is an interesting increase in improving the Banach contraction mapping in order to make the mapping become more general including the case that it is continuous or discontinuous. There is now extensive variety of literature dealing with fixed point problems via $\alpha$-admissible mappings (see [4, 14, 15, 19, 20]). The purpose of this paper is to do some work to improve and optimize the main results of [11] and [19]. Our conditions are much wider and more applicable. Further, an example is to highlight the superiority of the results obtained. Otherwise, an application in coping with the existence of a class of nonlinear integral equations is also presented.

We start our paper with some basic but necessary notions and terminologies as follows.

Definition 1.1 ([15]). Let $X$ be a nonempty set, and $\alpha: X \times X \rightarrow[0, \infty)$ and $f: X \rightarrow X$ be mappings. We say

(i) $f$ is an $\alpha$-admissible mapping if for all $x, y \in X, \alpha(x, y) \geq 1$ implies $\alpha(f x, f y) \geq 1$;

(ii) $X$ is $\alpha$-regular if $\left\{x_{n}\right\}$ is a sequence in $X$ such that $\alpha\left(x_{n}, x_{n+1}\right) \geq 1$ for all $n \in \mathbb{N}$ and $x_{n} \rightarrow x \in X$ as $n \rightarrow \infty$, then $\alpha\left(x_{n}, x\right) \geq 1$ for all $n \in \mathbb{N}$.

Definition $1.2([13,[16])$. A mapping $\varphi:[0, \infty) \rightarrow[0, \infty)$ is said to be an altering distance function if it holds:

(1) $\varphi$ is nondecreasing and continuous;

(2) $\varphi(t)=0$ if and only if $t=0$.

Theorem $1.3([15])$. Let $(X, d)$ be a complete metric space, $\varphi:[0, \infty) \rightarrow[0, \infty)$ be an altering distance function, and $f: X \rightarrow X$ be an $\alpha$-admissible mapping. Assume that $f$ is $\alpha$ - $\psi$-contractive mapping, that is,

$$
\alpha(x, y) d(f x, f y) \leq \psi(d(x, y)) .
$$

Also, suppose that the following conditions hold:

(i) there exists $x_{0} \in X$ such that $\alpha\left(x_{0}, f x_{0}\right) \geq 1$;

(ii) either $f$ is continuous or $X$ is $\alpha$-regular.

Then $f$ has a fixed point.

Remark 1.4 ([14]). If $\alpha(x, y)=1$ for all $x, y \in X$, then $f$ reduces into a Banach contraction. Notice that, in Theorem 1.3, $f$ is a weaker version of Banach contraction in the sense that $f$ does not necessarily satisfy the contractive condition (1.1) for all $x, y \in X$ (see Examples 2.3 and 2.4 of [15]).

Definition $1.5([20])$. Let $X$ be a nonempty set, and $\alpha: X \times X \rightarrow[0, \infty)$ and $f: X \rightarrow X$ be mappings. Then $f$ is said to be a weak $\alpha$-admissible mapping if for all $x \in X, \alpha(x, f x) \geq 1$, it follows that $\alpha(f x, f f x) \geq 1$.

Remark 1.6. It is customary to write $\mathcal{A}(X, \alpha)$ and $\mathcal{W} \mathcal{A}(X, \alpha)$ to denote the collection of all $\alpha$-admissible mappings on $X$ and the collection of all weak $\alpha$-admissible mappings on $X$. It may be verified that $\mathcal{A}(X, \alpha) \subseteq$ $\mathcal{W} \mathcal{A}(X, \alpha)$.

On the other hand, Banach contraction principle is dependent on the continuity of usual metric, that is, $d\left(x_{n}, y_{n}\right) \rightarrow d(x, y)$ as $x_{n} \rightarrow x$ and $y_{n} \rightarrow y$. This also brings us a limitation to utilize this principle. Fortunately, Bakhtin [2] or Czerwik [5] introduced the notion of $b$-metric space, or metric type space called by some authors, and they obtained more general assertions since $b$-metric is not necessarily continuous. Let us recall it as follows: 
Definition $1.7([2,5])$. Let $X$ be a nonempty set, $s \geq 1$ be a real number and $d: X \times X \rightarrow[0, \infty)$ be a mapping. We say that $d$ is a $b$-metric if for all $x, y, z \in X$, the following conditions are satisfied:

(d1) $d(x, y)=0$ if and only if $x=y$;

(d2) $d(x, y)=d(y, x)$;

(d3) $d(x, z) \leq s[d(x, y)+d(y, z)]$.

In this case, $(X, d)$ is called a $b$-metric space.

Definition $1.8([\underline{6}])$. Let $(X, d)$ be a $b$-metric space, $x \in X$ and $\left\{x_{n}\right\}$ a sequence in $X$. Then we say

(i) $\left\{x_{n}\right\} b$-converges to $x$ if $d\left(x_{n}, x\right) \rightarrow 0$ as $n \rightarrow \infty$. In this case, we write $\lim _{n \rightarrow \infty} x_{n}=x$.

(ii) $\left\{x_{n}\right\}$ is a $b$-Cauchy sequence if $d\left(x_{n}, x_{m}\right) \rightarrow 0$ as $n, m \rightarrow \infty$.

(iii) $(X, d)$ is $b$-complete if every $b$-Cauchy sequence is $b$-convergent in $X$.

(iv) A function $f: X \rightarrow Y$ is $b$-continuous at a point $x$ if $\left\{x_{n}\right\} b$-converges to $x$, then $\left\{f x_{n}\right\} b$-converges to $f x$, where $(Y, \rho)$ is a $b$-metric space.

Theorem $1.9([8])$. Let $(X, d)$ be a b-complete b-metric space with coefficient $s \geq 1$. Let $f: X \rightarrow X$ be a mapping. If there exists a real number $k \in\left[0, \frac{1}{s}\right)$ such that for all $x, y \in X$, the following inequality holds:

$$
d(f x, f y) \leq k d(x, y)
$$

then $f$ has a unique fixed point in $X$.

So far numerous authors have focused on fixed point results in $b$-metric spaces (see [1, 6, 17, 20]).

In the sequel, we always assume that $X$ is a nonempty set, $f: X \rightarrow X$ is a mapping. Write $\operatorname{Fix}(f)$ as the set of all fixed points of $f$ on $X$, that is, $\operatorname{Fix}(f):=\{x \in X \mid f x=x\}$. Also, for each elements $x$ and $y$ in a $b$-metric space $(X, d)$ with coefficient $s \geq 1$, let

$$
M_{s}(x, y):=\max \left\{d(x, y), d(x, f x), d(y, f y), \frac{d(x, f y)+d(y, f x)}{2 s}\right\},
$$

and

$$
N(x, y):=\min \{d(x, f x), d(y, f x)\} .
$$

Definition $1.10([19])$. Let $(X, d)$ be a $b$-metric space with coefficient $s \geq 1, \alpha: X \times X \rightarrow[0, \infty)$ and $\psi, \varphi:[0, \infty) \rightarrow[0, \infty)$ be given mappings. We say that a mapping $f: X \rightarrow X$ is an almost generalized $(\alpha, \psi, \varphi)_{s}$-contractive mapping if there exists $L \geq 0$ such that the following condition holds:

$$
x, y \in X \text { with } \alpha(x, y) \geq 1 \Longrightarrow \psi\left(s^{3} d(f x, f y)\right) \leq \psi\left(M_{s}(x, y)\right)-\varphi\left(M_{s}(x, y)\right)+L \psi(N(x, y)) .
$$

We denote with $\Xi_{s}(X, \alpha, \psi, \varphi)$ the collection of all almost generalized $(\alpha, \psi, \varphi)_{s}$-contractive mappings.

Theorem $1.11([19])$. Let $(X, d)$ be a $b$-complete $b$-metric space with coefficient $s \geq 1, \psi, \varphi:[0, \infty) \rightarrow[0, \infty)$ be altering distance functions, and $\alpha: X \times X \rightarrow[0, \infty)$ and $f: X \rightarrow X$ be two given mappings. Suppose that the following conditions hold:

$\left(A S_{1}\right) f \in \Xi_{s}(X, \alpha, \psi, \varphi) \cap \mathcal{W} \mathcal{A}(X, \alpha) ;$

$\left(A S_{2}\right)$ there exists $x_{0} \in X$ such that $\alpha\left(x_{0}, f x_{0}\right) \geq 1$; 
$\left(A S_{3}\right) \alpha$ has transitive property, that is, for $x, y, z \in X$,

$$
\alpha(x, y) \geq 1 \text { and } \alpha(y, z) \geq 1 \Rightarrow \alpha(x, z) \geq 1 ;
$$

$\left(A S_{4}\right) f$ is b-continuous.

Then $\operatorname{Fix}(f) \neq \emptyset$.

Theorem $1.12([19])$. Let $(X, d)$ be a b-complete $b$-metric space with coefficient $s \geq 1, \psi, \varphi:[0, \infty) \rightarrow[0, \infty)$ be two altering distance functions, and $\alpha: X \times X \rightarrow[0, \infty)$ and $f: X \rightarrow X$ be two given mappings. Suppose that the following conditions hold:

$\left(A S_{1}\right) f \in \Xi_{s}(X, \alpha, \psi, \varphi) \cap \mathcal{W} \mathcal{A}(X, \alpha) ;$

$\left(A S_{2}\right)$ there exists $x_{0} \in X$ such that $\alpha\left(x_{0}, f x_{0}\right) \geq 1$;

$\left(A S_{3}\right)$ a has transitive property;

$\left(\widetilde{A S_{4}}\right) X$ is $\alpha$-regular.

Then $\operatorname{Fix}(f) \neq \emptyset$.

Corollary 1.13 ([19]). Let $(X, d)$ be a b-complete b-metric space with coefficient $s \geq 1, \psi, \varphi:[0, \infty) \rightarrow[0, \infty)$ be altering distance functions, and $\alpha: X \times X \rightarrow[0, \infty)$ and $f: X \rightarrow X$ be two given mappings. Suppose that the following conditions hold:

$\left(\widetilde{A S_{1}}\right) f \in \Xi_{s}(X, \alpha, \psi, \varphi) \cap \mathcal{A}(X, \alpha) ;$

$\left(A S_{2}\right)$ there exists $x_{0} \in X$ such that $\alpha\left(x_{0}, f x_{0}\right) \geq 1$;

$\left(A S_{3}\right)$ a has transitive property;

$\left(A S_{4}\right) f$ is b-continuous.

Then $\operatorname{Fix}(f) \neq \emptyset$.

Corollary $1.14([19])$. Let $(X, d)$ be a $b$-complete b-metric space with coefficient $s \geq 1, \psi, \varphi:[0, \infty) \rightarrow[0, \infty)$ be two altering distance functions, and $\alpha: X \times X \rightarrow[0, \infty)$ and $f: X \rightarrow X$ be given mappings. Suppose that the following conditions hold:

$\left(\widetilde{A S_{1}}\right) f \in \Xi_{s}(X, \alpha, \psi, \varphi) \cap \mathcal{A}(X, \alpha) ;$

$\left(A S_{2}\right)$ there exists $x_{0} \in X$ such that $\alpha\left(x_{0}, f x_{0}\right) \geq 1$;

$\left(A S_{3}\right)$ a has transitive property;

$\left(\widetilde{A S_{4}}\right) X$ is $\alpha$-regular.

Then $\operatorname{Fix}(f) \neq \emptyset$.

Lemma 1.15 ([1]). Let $(X, d)$ be a b-metric space with coefficient $s \geq 1$ and let $\left\{x_{n}\right\}$ and $\left\{y_{n}\right\}$ be $b$ convergent to points $x, y \in X$, respectively. Then we have

$$
\frac{1}{s^{2}} d(x, y) \leq \liminf _{n \rightarrow \infty} d\left(x_{n}, y_{n}\right) \leq \limsup _{n \rightarrow \infty} d\left(x_{n}, y_{n}\right) \leq s^{2} d(x, y) .
$$

In particular, if $x=y$, then we have $\lim _{n \rightarrow \infty} d\left(x_{n}, y_{n}\right)=0$. Moreover, for each $z \in X$, we have

$$
\frac{1}{s} d(x, z) \leq \liminf _{n \rightarrow \infty} d\left(x_{n}, z\right) \leq \limsup _{n \rightarrow \infty} d\left(x_{n}, z\right) \leq s d(x, z) .
$$


Definition 1.16. Let $(X, d)$ be a $b$-metric space, $f: X \rightarrow X$, and $\alpha, \beta: X \times X \rightarrow[0, \infty)$ be given mappings. We say

(i) $f$ is an $(\alpha, \beta)$-admissible mapping if $\alpha(x, y) \geq 1$ and $\beta(x, y) \geq 1$ imply $\alpha(f x, f y) \geq 1$ and $\beta(f x, f y) \geq 1$ for all $x, y \in X$.

(ii) $X$ is $(\alpha, \beta)$-regular if $\left\{x_{n}\right\}$ is a sequence in $X$ such that $x_{n} \rightarrow x \in X, \alpha\left(x_{n}, x_{n+1}\right) \geq 1, \beta\left(x_{n}, x_{n+1}\right) \geq 1$, for all $n$, then there exists a subsequence $\left\{x_{n_{k}}\right\}$ of $\left\{x_{n}\right\}$ such that $\alpha\left(x_{n_{k}}, x\right) \geq 1, \beta\left(x_{n_{k}}, x\right) \geq 1$, for all $k \in \mathbb{N}$ and $\alpha(x, f x) \geq 1, \beta(x, f x) \geq 1$.

Definition 1.17 ([1] $)$. Let $(X, d)$ be a $b$-metric space, $\psi:[0, \infty) \rightarrow[0, \infty)$ be an altering distance function, and $\alpha, \beta: X \times X \rightarrow[0, \infty)$ be given mappings. A mapping $f: X \rightarrow X$ is said to be $(\alpha, \beta)$-Geraghty type contractive mapping if there exists a function $\theta \in \Theta$, such that for all $x, y \in X$, the following condition holds:

$$
\alpha(x, f x) \beta(y, f y) \psi\left(s^{3} d(f x, f y)\right) \leq \theta\left(\psi\left(M_{s}(x, y)\right)\right) \psi\left(M_{s}(x, y)\right),
$$

where $\Theta$ is a family of functions $\theta:[0, \infty) \rightarrow[0,1)$ such that for any bounded positive real sequence $\left\{t_{n}\right\}$, $\theta\left(t_{n}\right) \rightarrow 1$ implies $t_{n} \rightarrow 0$.

Theorem 1.18 ([11]). Let $(X, d)$ be a b-complete b-metric space, $f: X \rightarrow X$, and $\alpha, \beta: X \times X \rightarrow[0, \infty)$ be given mappings. Suppose that the following conditions hold:

(i) $f$ is an $(\alpha, \beta)$-admissible mapping;

(ii) $f$ is an $(\alpha, \beta)$-Geraghty type contractive mapping;

(iii) there exists $x_{0} \in X$ such that $\alpha\left(x_{0}, f x_{0}\right) \geq 1$ and $\beta\left(x_{0}, f x_{0}\right) \geq 1$;

(iv) either $f$ is b-continuous or $X$ is $(\alpha, \beta)$-regular.

Then $f$ has a unique fixed point.

Remark 1.19. (ii) of Definition 1.16 corrects and improves Definition 4.2 of [11] (see also Definition 1.3 of [4]). In fact, Definition 4.2 of [11] has some problems. Since $\alpha\left(x_{n}, x_{n+1}\right) \geq 1$ and $\beta\left(x_{n}, x_{n+1}\right) \geq 1$ for all $n \in \mathbb{N}$, then clearly, $\alpha\left(x_{n_{k}}, x_{n_{k}+1}\right) \geq 1$ and $\beta\left(x_{n_{k}}, x_{n_{k}+1}\right) \geq 1$ for all $k \in \mathbb{N}$. Hence both Theorem 4.4 of [11] and Theorem 2.1 of [4] made some mistakes.

\section{Main results}

In this section, we introduce some concepts which greatly improve Definitions 1.10 and 1.17. Based on them, we obtain some fixed point theorems with simple conditions and vivid proofs, which, to the best of our knowledge, are new. Moreover, we give an example to illustrate the advantage of our results.

Definition 2.1. Let $(X, d)$ be a $b$-metric space with coefficient $s \geq 1$. Let $\varepsilon>1$ be a constant and $\alpha: X \times X \rightarrow[0, \infty)$ and $\psi:[0, \infty) \rightarrow[0, \infty)$ be given mappings. We say that a mapping $f: X \rightarrow X$ is an almost generalized $(\alpha, \psi)_{s}$-contractive mapping if there exists $L \geq 0$ such that the following condition holds:

$$
x, y \in X \text { with } \alpha(x, y) \geq 1 \Longrightarrow \psi\left(s^{\varepsilon} d(f x, f y)\right) \leq \psi\left(M_{s}(x, y)\right)+L \psi(N(x, y)) .
$$

In this case, write $\Xi_{s}(X, \alpha, \psi)$ as the collection of all almost generalized $(\alpha, \psi)_{s^{-}}$contractive mappings.

Theorem 2.2. Let $(X, d)$ be a b-complete b-metric space with coefficient $s>1, \psi:[0, \infty) \rightarrow[0, \infty)$ be an altering distance function and $\alpha: X \times X \rightarrow[0, \infty)$ and $f: X \rightarrow X$ be two given mappings. Suppose that the following conditions hold: 
(1) $f \in \Xi_{s}(X, \alpha, \psi) \cap \mathcal{W} \mathcal{A}(X, \alpha)$;

(2) there exists $x_{0} \in X$ such that $\alpha\left(x_{0}, f x_{0}\right) \geq 1$;

(3) $f$ is b-continuous.

Then $\operatorname{Fix}(f) \neq \emptyset$.

Proof. By (2), for $x_{0} \in X$, construct a sequence $\left\{x_{n}\right\}$ with $x_{n+1}=f x_{n}, n \in \mathbb{N}$. Assume that $x_{n_{0}}=x_{n_{0}+1}$ for some $n_{0}$, then $\operatorname{Fix}(f)=\left\{x_{n_{0}}\right\}$, in this case, the proof is valid. Without loss of generality, put $x_{n} \neq x_{n+1}$ for all $n$. We begin by proving the following inequality:

$$
d\left(x_{n+1}, x_{n+2}\right) \leq \lambda d\left(x_{n}, x_{n+1}\right),
$$

where $\lambda \in\left[0, \frac{1}{s}\right)$ is a constant.

On account of $f \in \mathcal{W} \mathcal{A}(X, \alpha)$ and $\alpha\left(x_{0}, f x_{0}\right) \geq 1$, it follows that

$$
\alpha\left(x_{1}, x_{2}\right)=\alpha\left(f x_{0}, f f x_{0}\right) \geq 1, \quad \alpha\left(x_{2}, x_{3}\right)=\alpha\left(f x_{1}, f f x_{1}\right) \geq 1 .
$$

Using this process again, we get

$$
\alpha\left(x_{n}, x_{n+1}\right) \geq 1
$$

for all $n$. By 2.1, one has

$$
\begin{aligned}
\psi\left(s^{\varepsilon} d\left(x_{n+1}, x_{n+2}\right)\right) & =\psi\left(s^{\varepsilon} d\left(f x_{n}, f x_{n+1}\right)\right) \\
& \leq \psi\left(M_{s}\left(x_{n}, x_{n+1}\right)\right)+L \psi\left(N\left(x_{n}, x_{n+1}\right)\right)
\end{aligned}
$$

where

$$
\begin{aligned}
M_{s}\left(x_{n}, x_{n+1}\right) & =\max \left\{d\left(x_{n}, x_{n+1}\right), d\left(x_{n}, f x_{n}\right), d\left(x_{n+1}, f x_{n+1}\right), \frac{d\left(x_{n}, f x_{n+1}\right)+d\left(x_{n+1}, f x_{n}\right)}{2 s}\right\} \\
& =\max \left\{d\left(x_{n}, x_{n+1}\right), d\left(x_{n}, x_{n+1}\right), d\left(x_{n+1}, x_{n+2}\right), \frac{d\left(x_{n}, x_{n+2}\right)+d\left(x_{n+1}, x_{n+1}\right)}{2 s}\right\} \\
& \left.\leq \max \left\{d\left(x_{n}, x_{n+1}\right), d\left(x_{n+1}, x_{n+2}\right), \frac{d\left(x_{n}, x_{n+1}\right)+d\left(x_{n+1}, x_{n+2}\right)}{2}\right)\right\} \\
& =\max \left\{d\left(x_{n}, x_{n+1}\right), d\left(x_{n+1}, x_{n+2}\right)\right\},
\end{aligned}
$$

and

$$
\begin{aligned}
N\left(x_{n}, x_{n+1}\right) & =\min \left\{d\left(x_{n}, f x_{n}\right), d\left(x_{n+1}, f x_{n}\right)\right\} \\
& =\min \left\{d\left(x_{n}, x_{n+1}\right), d\left(x_{n+1}, x_{n+1}\right)\right\} \\
& =\min \left\{d\left(x_{n}, x_{n+1}\right), 0\right\} \\
& =0 .
\end{aligned}
$$

Making the most of (2.3), 2.4 , 2.5), and Definition 1.2, we arrive at

$$
s^{\varepsilon} d\left(x_{n+1}, x_{n+2}\right) \leq \max \left\{d\left(x_{n}, x_{n+1}\right), d\left(x_{n+1}, x_{n+2}\right)\right\} .
$$

If $d\left(x_{n}, x_{n+1}\right) \leq d\left(x_{n+1}, x_{n+2}\right)$ for some $n \in \mathbb{N}$, then 2.6 follows that

$$
s^{\varepsilon} d\left(x_{n+1}, x_{n+2}\right) \leq d\left(x_{n+1}, x_{n+2}\right) .
$$

Thus, $d\left(x_{n+1}, x_{n+2}\right)=0$, it is a contradiction. If $d\left(x_{n+1}, x_{n+2}\right) \leq d\left(x_{n}, x_{n+1}\right)$ for all $n \in \mathbb{N}$, then (2.6) gives that

$$
s^{\varepsilon} d\left(x_{n+1}, x_{n+2}\right) \leq d\left(x_{n}, x_{n+1}\right) .
$$


Accordingly, 2.2 holds, where $\lambda=\frac{1}{s^{\varepsilon}} \in\left[0, \frac{1}{s}\right)$.

Now via Theorem 1.9 or Lemma 3.1 of [8], making full use of 2.2 , we infer that $\left\{x_{n}\right\}$ is a $b$-Cauchy sequence. Since $(X, d)$ is $b$-complete, we claim that $\left\{x_{n}\right\} b$-converges to some point $x^{*} \in X$. Finally, we show $x^{*} \in \operatorname{Fix}(f)$. Indeed, by using (3), it is not hard to verify that

$$
d\left(f x^{*}, x^{*}\right) \leq s\left[d\left(f x^{*}, f x_{n}\right)+d\left(f x_{n}, x^{*}\right)\right]=s\left[d\left(f x^{*}, f x_{n}\right)+d\left(x_{n+1}, x^{*}\right)\right] \rightarrow 0 \text { as } n \rightarrow \infty .
$$

Consequently, $d\left(f x^{*}, x^{*}\right)=0$, i.e., $x^{*} \in \operatorname{Fix}(f)$.

Theorem 2.3. Let $(X, d)$ be a b-complete $b$-metric space with coefficient $s>1, \psi:[0, \infty) \rightarrow[0, \infty)$ be an altering distance function and $\alpha: X \times X \rightarrow[0, \infty)$ and $f: X \rightarrow X$ be two given mappings. Suppose that the following conditions hold:

(1) $f \in \Xi_{s}(X, \alpha, \psi) \cap \mathcal{W} \mathcal{A}(X, \alpha)$;

(2) there exists $x_{0} \in X$ such that $\alpha\left(x_{0}, f x_{0}\right) \geq 1$;

(3) $X$ is $\alpha$-regular.

Then $\operatorname{Fix}(f) \neq \emptyset$.

Proof. Repeating the previous argument in Theorem 2.2, we construct a sequence $\left\{x_{n}\right\}$ satisfying $x_{n+1}=$ $f x_{n} \rightarrow x^{*} \in X$ as $n \rightarrow \infty$. Then by (3), we get $\alpha\left(x_{n}, x^{*}\right) \geq 1$ for all $n \in \mathbb{N}$. In view of (1), we speculate

$$
\psi\left(s^{\varepsilon} d\left(f x_{n}, f x^{*}\right)\right) \leq \psi\left(M_{s}\left(x_{n}, x^{*}\right)\right)+L \psi\left(N\left(x_{n}, x^{*}\right)\right) .
$$

Since $\psi$ is continuous, then by (2.7), it is easy to see that

$$
\psi\left(s^{\varepsilon} \lim _{n \rightarrow \infty} d\left(f x_{n}, f x^{*}\right)\right) \leq \psi\left(\lim _{n \rightarrow \infty} M_{s}\left(x_{n}, x^{*}\right)\right)+L \psi\left(\lim _{n \rightarrow \infty} N\left(x_{n}, x^{*}\right)\right) .
$$

Note that

$$
\begin{aligned}
M_{s}\left(x_{n}, x^{*}\right) & =\max \left\{d\left(x_{n}, x^{*}\right), d\left(x_{n}, f x_{n}\right), d\left(x^{*}, f x^{*}\right), \frac{d\left(x_{n}, f x^{*}\right)+d\left(x^{*}, f x_{n}\right)}{2 s}\right\} \\
& \leq \max \left\{d\left(x_{n}, x^{*}\right), s\left[d\left(x_{n}, x^{*}\right)+d\left(x_{n+1}, x^{*}\right)\right], d\left(x^{*}, f x^{*}\right), \frac{d\left(x_{n}, x^{*}\right)+d\left(x^{*}, f x^{*}\right)}{2}+\frac{d\left(x^{*}, x_{n+1}\right)}{2 s}\right\} \\
& \rightarrow \max \left\{0,0, d\left(x^{*}, f x^{*}\right), \frac{d\left(x^{*}, f x^{*}\right)}{2}\right\}=d\left(x^{*}, f x^{*}\right)(n \rightarrow \infty),
\end{aligned}
$$

it follows that

$$
\lim _{n \rightarrow \infty} M_{s}\left(x_{n}, x^{*}\right) \leq d\left(x^{*}, f x^{*}\right)
$$

Moreover, we deduce that

$$
\begin{aligned}
N\left(x_{n}, x^{*}\right) & =\min \left\{d\left(x_{n}, f x_{n}\right), d\left(x^{*}, f x_{n}\right)\right\} \\
& =\min \left\{d\left(x_{n}, x_{n+1}\right), d\left(x^{*}, x_{n+1}\right)\right\} \\
& \rightarrow \min \{0,0\}=0(n \rightarrow \infty),
\end{aligned}
$$

which establishes that

$$
\lim _{n \rightarrow \infty} N\left(x_{n}, x^{*}\right)=0 .
$$

Making the most of Definition 1.2 and 2.8, 2.9 and (2.10), we demonstrate that

$$
s^{\varepsilon} \lim _{n \rightarrow \infty} d\left(f x_{n}, f x^{*}\right) \leq d\left(x^{*}, f x^{*}\right),
$$


which implies that

$$
\lim _{n \rightarrow \infty} d\left(f x_{n}, f x^{*}\right) \leq \frac{1}{s^{\varepsilon}} d\left(x^{*}, f x^{*}\right) .
$$

Notice that

$$
\frac{1}{s} d\left(x^{*}, f x^{*}\right) \leq d\left(x^{*}, f x_{n}\right)+d\left(f x_{n}, f x^{*}\right)=d\left(x^{*}, x_{n+1}\right)+d\left(f x_{n}, f x^{*}\right) .
$$

Taking the limit as inequality 2.12 and utilizing (2.11), we obtain

$$
\frac{1}{s} d\left(x^{*}, f x^{*}\right) \leq \frac{1}{s^{\varepsilon}} d\left(x^{*}, f x^{*}\right),
$$

which means that $d\left(x^{*}, f x^{*}\right)=0$, that is, $x^{*} \in \operatorname{Fix}(f)$.

Corollary 2.4. Let $(X, d)$ be a b-complete $b$-metric space with coefficient $s>1, \psi:[0, \infty) \rightarrow[0, \infty)$ be an altering distance function, and $\alpha: X \times X \rightarrow[0, \infty)$ and $f: X \rightarrow X$ be two given mappings. Suppose that the following conditions hold:

(1) $f \in \Xi_{s}(X, \alpha, \psi) \cap \mathcal{A}(X, \alpha)$;

(2) there exists $x_{0} \in X$ such that $\alpha\left(x_{0}, f x_{0}\right) \geq 1$;

(3) $f$ is b-continuous.

Then $\operatorname{Fix}(f) \neq \emptyset$.

Corollary 2.5. Let $(X, d)$ be a b-complete b-metric space with coefficient $s>1, \psi:[0, \infty) \rightarrow[0, \infty)$ be an altering distance function, and $\alpha: X \times X \rightarrow[0, \infty)$ and $f: X \rightarrow X$ be two given mappings. Suppose that the following conditions hold:

(1) $f \in \Xi_{s}(X, \alpha, \psi) \cap \mathcal{A}(X, \alpha)$;

(2) there exists $x_{0} \in X$ such that $\alpha\left(x_{0}, f x_{0}\right) \geq 1$;

(3) $X$ is $\alpha$-regular.

Then $\operatorname{Fix}(f) \neq \emptyset$.

Remark 2.6. Theorems 2.2, 2.3 and Corollaries 2.4, 2.5 greatly optimize and improve Theorems $1.11,1.12$ and Corollaries 1.13, 1.14, respectively. Indeed, on the one hand, compared with (1.2), (2.1) deletes the item $\varphi\left(M_{s}(x, y)\right)$ which makes the inequality become much wider. This is a great improvement. Likewise, our condition is much more general because the index $\varepsilon>1$ is arbitrary. Evidently, $\varepsilon=3$ is the special case of $\varepsilon>1$. On the other hand, our results dispense with the condition of transitive property of the mapping $\alpha$. Needless to say, the conditions of our theorems are weaker than Sintunavarat's theorems. Therefore, our conclusions may be more convenient than Sintunavarat's in applications.

Remark 2.7. From the proofs of our theorems, it is easy to see that we do not use Lemma 1.15. Our proofs are shorter since we need not consider the $b$-discontinuity of $b$-metric. Whereas, in order to overcome the difficulty of the $b$-discontinuity of $b$-metric, the proofs of Sintunavarat's theorems are very complicated based on the fact of depending on Lemma 1.15 strongly.

Example 2.8. Let $X=\mathbb{R}$ and define

$$
d(x, y)=|x-y|^{2}
$$

for all $x, y \in X$. Then $(X, d)$ is a $b$-complete $b$-metric space with coefficient $s=2$. Define mappings 
$f: X \rightarrow X$ and $\alpha: X \times X \rightarrow[0, \infty)$ by

$$
f x= \begin{cases}\frac{x}{3}, & x \in[0,5], \\ \frac{1}{6} x+\frac{5}{6}, & x \in(5, \infty),\end{cases}
$$

and

$$
\alpha(x, y)= \begin{cases}\frac{2}{3}+\frac{1}{|x-y|}, & x, y \in[0,5] \\ 0, & \text { otherwise. }\end{cases}
$$

Let us first prove $f \in \Xi_{s}(X, \alpha, \psi)$. Assume that $x, y \in X$ with $\alpha(x, y) \geq 1$ and hence $x, y \in[0,5]$ with $|x-y| \leq 3$. Let $1<\varepsilon \leq 3$ and $L \geq 0$ be constants and $\psi(t)=k t(k>0)$. Then

$$
\begin{aligned}
\psi\left(s^{\varepsilon} d(f x, f y)\right) & =\psi\left(2^{\varepsilon}|f x-f y|^{2}\right) \\
& =\psi\left(2^{\varepsilon}\left|\frac{x}{3}-\frac{y}{3}\right|^{2}\right) \\
& \leq \psi\left(2^{\varepsilon-3}|x-y|^{2}\right) \\
& =\psi\left(2^{\varepsilon-3} d(x, y)\right) \\
& \leq \psi(d(x, y)) \\
& \leq \psi\left(M_{s}(x, y)\right)+L \psi(N(x, y)) .
\end{aligned}
$$

As a consequence, $f \in \Xi_{s}(X, \alpha, \psi)$.

Likewise, we prove $f \in \mathcal{W} \mathcal{A}_{s}(X, \alpha)$. Indeed, if $x \in X$ and $\alpha(x, f x) \geq 1$, then $x, f x \in[0,5]$ and $|x-f x| \leq 3$. Thus, $x \in\left[0, \frac{9}{2}\right]$. This indicates that $f f x \in\left[0, \frac{1}{2}\right]$ and hence

$$
\alpha(f x, f f x)=\frac{2}{3}+\frac{9}{2|x|} \geq \frac{5}{3} \geq 1 .
$$

Otherwise, obviously $f$ is $b$-continuous and there exists $x_{0}=1$ such that

$$
\alpha\left(x_{0}, f x_{0}\right)=\alpha(1, f 1)=\frac{2}{3}+\frac{1}{|1-f 1|}=\frac{13}{6} \geq 1 .
$$

Consequently, all conditions of Theorem 2.2 hold. So $\operatorname{Fix}(f)=\{0\} \neq \emptyset$.

However, we cannot use Theorem 1.11 to get $\operatorname{Fix}(f) \neq \emptyset$, since $\alpha$ does not satisfy condition $\left(A S_{3}\right)$ of this theorem. Indeed, put $x=5, y=4, z=1$. Now that $\alpha(x, y)=\frac{5}{3} \geq 1$ and $\alpha(y, z)=1 \geq 1$, but $\alpha(x, z)=\frac{11}{12}<1$. Then $\left(A S_{3}\right)$ does not hold in this example. In other words, Theorem 2.2 is more superior than Theorem 1.11 .

Definition 2.9. Let $(X, d)$ be a $b$-metric space, $\psi:[0, \infty) \rightarrow[0, \infty)$ be an altering distance function, and $\alpha, \beta: X \times X \rightarrow[0, \infty)$ be given mappings. A mapping $f: X \times X$ is said to be weak $(\alpha, \beta)$-Geraghty type contractive mapping if there exists $\varepsilon>1$ such that the following condition holds:

$$
x, y \in X \text { with } \alpha(x, y) \geq 1 \text { and } \beta(x, y) \geq 1 \Longrightarrow \alpha(x, f x) \beta(y, f y) \psi\left(s^{\varepsilon} d(f x, f y)\right) \leq \psi\left(M_{s}(x, y)\right) .
$$

Theorem 2.10. Let $(X, d)$ be a b-complete b-metric space, $f: X \rightarrow X$, and $\alpha, \beta: X \times X \rightarrow[0, \infty)$ be given mappings. Suppose that the following conditions hold:

(i) $f$ is an $(\alpha, \beta)$-admissible mapping;

(ii) $f$ is a weak $(\alpha, \beta)$-Geraghty type contractive mapping;

(iii) there exists $x_{0} \in X$ such that $\alpha\left(x_{0}, f x_{0}\right) \geq 1$ and $\beta\left(x_{0}, f x_{0}\right) \geq 1$;

(iv) either $f$ is b-continuous or $X$ is $(\alpha, \beta)$-regular. 
Then $f$ has a fixed point $x^{*} \in X$. Moreover, if $X$ is $(\alpha, \beta)$-regular and for $x, y \in X, \alpha(x, x) \geq 1$ and $\beta(y, y) \geq 1$ imply $\alpha(x, y) \geq 1$ and $\beta(x, y) \geq 1$, then the fixed point is unique.

Proof. By (iii), for $x_{0} \in X$, construct a sequence $\left\{x_{n}\right\}$ satisfying $x_{n+1}=f x_{n}, n \in \mathbb{N}$. Assume that $x_{n_{0}}=x_{n_{0}+1}$ for some $n_{0}$, then $\operatorname{Fix}(f)=\left\{x_{n_{0}}\right\} \neq \emptyset$, in this case, the conclusion is satisfied. So set $x_{n} \neq x_{n+1}$ for all $n$, that is, $d\left(x_{n}, x_{n+1}\right)>0$ for all $n$. By (i),

$$
\alpha\left(x_{0}, f x_{0}\right)=\alpha\left(x_{0}, x_{1}\right) \geq 1 \Rightarrow \alpha\left(f x_{0}, f x_{1}\right)=\alpha\left(x_{1}, x_{2}\right) \geq 1 \Rightarrow \alpha\left(f x_{1}, f x_{2}\right)=\alpha\left(x_{2}, x_{3}\right) \geq 1 .
$$

Continuing this process, we get $\alpha\left(x_{n}, x_{n+1}\right) \geq 1$ for all $n \in \mathbb{N}$. Similarly we get $\beta\left(x_{n}, x_{n+1}\right) \geq 1$ for all $n \in \mathbb{N}$. From (2.14), we have

$$
\begin{aligned}
\psi\left(s^{\varepsilon} d\left(x_{n+1}, x_{n+2}\right)\right) & =\psi\left(s^{\varepsilon} d\left(f x_{n}, f x_{n+1}\right)\right) \\
& \leq \alpha\left(x_{n}, f x_{n}\right) \beta\left(x_{n+1}, f x_{n+1}\right) \psi\left(s^{\varepsilon} d\left(f x_{n}, f x_{n+1}\right)\right) \\
& \leq \psi\left(M_{s}\left(x_{n}, x_{n+1}\right)\right)
\end{aligned}
$$

which follows that

$$
s^{\varepsilon} d\left(x_{n+1}, x_{n+2}\right) \leq M_{s}\left(x_{n}, x_{n+1}\right) .
$$

By using (2.4), we get 2.6). Following an argument similar to that given in Theorem 2.2, we conclude that $\left\{x_{n}\right\} b$-converges to some point $x^{*} \in X$. Finally, we show $x^{*} \in \operatorname{Fix}(f)$.

Actually, if $f$ is $b$-continuous, then

$$
d\left(f x^{*}, x^{*}\right) \leq s\left[d\left(f x^{*}, f x_{n}\right)+d\left(f x_{n}, x^{*}\right)\right]=s\left[d\left(f x^{*}, f x_{n}\right)+d\left(x_{n+1}, x^{*}\right)\right] \rightarrow 0 \text { as } n \rightarrow \infty .
$$

Accordingly, $d\left(f x^{*}, x^{*}\right)=0$, that is to say, $x^{*} \in \operatorname{Fix}(f)$.

If $X$ is $(\alpha, \beta)$-regular, then there exists a subsequence $\left\{x_{n_{k}}\right\}$ of $\left\{x_{n}\right\}$ such that $\alpha\left(x_{n_{k}}, x^{*}\right) \geq 1$ and $\beta\left(x_{n_{k}}, x^{*}\right) \geq 1$ for all $k \in \mathbb{N}$ and $\alpha\left(x^{*}, f x^{*}\right) \geq 1$ and $\beta\left(x^{*}, f x^{*}\right) \geq 1$. Now from 2.14, we have that

$$
\begin{aligned}
\psi\left(s^{\varepsilon} d\left(f x_{n_{k}}, f x^{*}\right)\right) & \leq \alpha\left(x_{n_{k}}, f x_{n_{k}}\right) \beta\left(x^{*}, f x^{*}\right) \psi\left(s^{\varepsilon} d\left(f x_{n_{k}}, f x^{*}\right)\right) \\
& \leq \psi\left(M_{s}\left(x_{n_{k}}, x^{*}\right)\right) .
\end{aligned}
$$

By the monotonicity of the function $\psi$, one can see that

$$
\begin{aligned}
& s^{\varepsilon} d\left(f x_{n_{k}}, f x^{*}\right) \leq M_{s}\left(x_{n_{k}}, x^{*}\right) \\
& =\max \left\{d\left(x_{n_{k}}, x^{*}\right), d\left(x_{n_{k}}, f x_{n_{k}}\right), d\left(x^{*}, f x^{*}\right), \frac{d\left(x_{n_{k}}, f x^{*}\right)+d\left(x^{*}, f x_{n_{k}}\right)}{2 s}\right\} \\
& \leq \max \left\{d\left(x_{n_{k}}, x^{*}\right), s\left[d\left(x_{n_{k}}, x^{*}\right)+d\left(x_{n_{k}+1}, x^{*}\right)\right], d\left(x^{*}, f x^{*}\right)\right. \\
& \left., \frac{d\left(x_{n_{k}}, x^{*}\right)+d\left(x^{*}, f x^{*}\right)}{2}+\frac{d\left(x^{*}, x_{n_{k}+1}\right)}{2 s}\right\} \\
& \rightarrow \max \left\{0,0, d\left(x^{*}, f x^{*}\right), \frac{d\left(x^{*}, f x^{*}\right)}{2}\right\}=d\left(x^{*}, f x^{*}\right)(k \rightarrow \infty),
\end{aligned}
$$

which implies that

$$
\lim _{k \rightarrow \infty} d\left(f x_{n_{k}}, f x^{*}\right) \leq \frac{1}{s^{\varepsilon}} d\left(x^{*}, f x^{*}\right) .
$$

Note that

$$
\frac{1}{s} d\left(x^{*}, f x^{*}\right) \leq d\left(x^{*}, f x_{n_{k}}\right)+d\left(f x_{n_{k}}, f x^{*}\right)=d\left(x^{*}, x_{n_{k}+1}\right)+d\left(f x_{n_{k}}, f x^{*}\right) .
$$


Equations 2.15) and 2.16) give that

$$
\frac{1}{s} d\left(x^{*}, f x^{*}\right) \leq \frac{1}{s^{\varepsilon}} d\left(x^{*}, f x^{*}\right),
$$

which leads to $d\left(x^{*}, f x^{*}\right)=0$, that is, $x^{*} \in \operatorname{Fix}(f)$.

After having finished proving $x^{*} \in \operatorname{Fix}(f)$, if for $x, y \in X, \alpha(x, x) \geq 1$ and $\beta(x, x) \geq 1$ imply $\alpha(x, y) \geq 1$ and $\beta(x, y) \geq 1$, let us prove the uniqueness of fixed point of $f$ provided that $X$ is $(\alpha, \beta)$-regular. To this end, we suppose for absurd that there is another fixed point $y^{*}$, then $\alpha\left(x^{*}, f x^{*}\right) \geq 1$ and $\beta\left(y^{*}, f y^{*}\right) \geq 1$. So $\alpha\left(x^{*}, x^{*}\right) \geq 1$ and $\beta\left(y^{*}, y^{*}\right) \geq 1$. In view of the hypothesis, we have $\alpha\left(x^{*}, y^{*}\right) \geq 1$ and $\beta\left(x^{*}, y^{*}\right) \geq 1$. Now by applying (2.14, we arrive at

$$
\begin{aligned}
\psi\left(s^{\varepsilon} d\left(f x^{*}, f y^{*}\right)\right) & \leq \alpha\left(x^{*}, f x^{*}\right) \beta\left(y^{*}, f y^{*}\right) \psi\left(s^{\varepsilon} d\left(f x^{*}, f y^{*}\right)\right) \\
& \leq \psi\left(M_{s}\left(x^{*}, y^{*}\right)\right) .
\end{aligned}
$$

By the monotonicity of the function $\psi$, we conclude that

$$
\begin{aligned}
s^{\varepsilon} d\left(f x^{*}, f y^{*}\right) & \leq M_{s}\left(x^{*}, y^{*}\right) \\
& =\max \left\{d\left(x^{*}, y^{*}\right), d\left(x^{*}, f x^{*}\right), d\left(y^{*}, f y^{*}\right), \frac{d\left(x^{*}, f y^{*}\right)+d\left(y^{*}, f x^{*}\right)}{2 s}\right\} \\
& =\max \left\{d\left(x^{*}, y^{*}\right), 0,0, \frac{d\left(x^{*}, y^{*}\right)}{s}\right\} \\
& =d\left(x^{*}, y^{*}\right),
\end{aligned}
$$

which means that $d\left(x^{*}, y^{*}\right)=0$. Therefore, $x^{*}=y^{*}$.

Remark 2.11. Theorem 2.10 is much superior in several aspects as compared to Theorem 1.18 . Indeed, (2.14) dismisses the condition $\theta\left(\psi\left(M_{s}(x, y)\right)\right)$ of (1.3). This indicates that (2.14) is much broader than (1.3). Furthermore, the constant $\varepsilon>1$ is much more general in 2.14 because it is not only restricted to $\varepsilon=3$ in 1.3. Likewise, in 2.14, $x, y \in X$ are selected to satisfy the condition that $\alpha(x, y) \geq 1$ and $\beta(x, y) \geq 1$, whereas in $(1.3), x, y \in X$ are arbitrary. This implies that (2.14) is weaker than (1.3). Otherwise, the proof of Theorem 2.10 is simpler than the one of Theorem 1.18 because it ignores Lemma 1.15. but Theorem 1.18 depends on this lemma utterly (in fact, in the proof of Theorem 1.18 this lemma was shown and used indirectly).

Remark 2.12. Throughout the whole proof of Theorem 1.18 (that is, Theorem 4.4 of [11], the proof of the uniqueness of fixed point is incorrect. This is because the authors from [11] increased some additional conditions such as $\alpha\left(x^{*}, T x^{*}\right) \geq 1, \alpha\left(y^{*}, T y^{*}\right) \geq 1, \beta\left(x^{*}, T x^{*}\right) \geq 1$, and $\beta\left(y^{*}, T y^{*}\right) \geq 1$ when $T$ is continuous, whereas these conditions hold only if $X$ is $(\alpha, \beta)$-regular. However, these conditions did not appear in the hypotheses of Theorem 1.18. Hence, the proof is false if $T$ is continuous. The proof of Theorem 2.10 notes the above problems and shows the superiority.

\section{Application}

In this section, we prove the existence theorem for a solution of the following integral equation by using our main results in the previous section:

$$
x(c)=\Omega(\phi(c), c)+K(c, c, \phi(c))+\int_{a}^{b} K(c, r, x(r)) \mathrm{d} r,
$$

where $a, b \in \mathbb{R}, x \in C[a, b]$ (the set of all continuous functions defined on $[a, b]$ ), $\phi:[a, b] \rightarrow \mathbb{R}, \Omega: \mathbb{R} \times[a, b] \rightarrow$ $\mathbb{R}$ and $K:[a, b] \times[a, b] \times \mathbb{R} \rightarrow \mathbb{R}$ are given mappings.

The following theorem greatly improves Theorem 3.1] of [19] with simpler conditions, which emphasizes the superiority of our results. 
Theorem 3.1. Consider the integral equation (3.1). Suppose that the following conditions hold:

(i) $K:[a, b] \times[a, b] \times \mathbb{R} \rightarrow \mathbb{R}$ is continuous and nondecreasing in the third order;

(ii) there exists $p>1$ satisfying the following condition: for each $r, c \in[a, b]$ and $x, y \in C[a, b]$ with $x(w) \leq y(w)$ for all $w \in[a, b]$, we have

$$
|K(c, r, x(r))-K(c, r, y(r))| \leq \xi(c, r)|x(r)-y(r)|,
$$

where $\xi:[a, b] \times[a, b] \rightarrow[0, \infty)$ is a continuous function satisfying

$$
\sup _{c \in[a, b]}\left(\int_{a}^{b} \xi(c, r)^{p} \mathrm{~d} r\right) \leq \frac{1}{2^{\varepsilon p-\varepsilon}(b-a)^{p-1}}
$$

and $\varepsilon>1$ is a constant;

(iii) there exists $x_{0} \in C[a, b]$ such that $x_{0}(c) \leq \Omega(\phi(c), c)+K(c, c, \phi(c))+\int_{a}^{b} K\left(c, r, x_{0}(r)\right) \mathrm{d} r$ for all $c \in[a, b]$.

Then the integral equation (3.1) has a solution.

Proof. Put $X=C[a, b]$ and define a mapping $f: X \rightarrow X$ by

$$
(f x)(c)=\Omega(\phi(c), c)+K(c, c, \phi(c))+\int_{a}^{b} K(c, r, x(r)) \mathrm{d} r
$$

for all $x \in X$ and $c \in[a, b]$. Define a mapping $d: X \times X \rightarrow[0, \infty)$ by

$$
d(x, y)=\sup _{c \in[a, b]}|x(c)-y(c)|^{p} \quad(p>1),
$$

for all $x, y \in X$. Clearly, $(X, d)$ is a $b$-complete $b$-metric space with coefficient $s=2^{p-1}>1$. Next, we define a mapping $\alpha: X \times X \rightarrow[0, \infty)$ by

$$
\alpha(x, y)= \begin{cases}1, & x(c) \leq y(c) \text { for all } c \in[a, b] \\ \lambda, & \text { otherwise }\end{cases}
$$

where $0<\lambda<1$. Since $K$ is nondecreasing in the third order, we get $f \in \mathcal{A}(X, \alpha) \subset \mathcal{W} \mathcal{A}(X, \alpha)$. By (iii), (2) in Theorem 2.3 is satisfied. Also, we get that condition (3) in Theorem 2.3 holds (see [10]).

In the end, we prove $f \in \Xi_{s}(X, \alpha, \psi)$. To this end, we choose $q \in \mathbb{R}$ such that $\frac{1}{p}+\frac{1}{q}=1$. Let $x, y \in X$ satisfy $\alpha(x, y) \geq 1$, that is, $x(c) \leq y(c)$ for all $c \in[a, b]$. Let $\psi(t)=t$ and $L \geq 0$ be a constant. The fact (ii) along with the Hölder inequality yield that

$$
\begin{aligned}
2^{\varepsilon p-\varepsilon}|(f x)(c)-(f y)(c)|^{p} & \leq 2^{\varepsilon p-\varepsilon}\left(\int_{a}^{b}|K(c, r, x(r))-K(c, r, y(r))| \mathrm{d} r\right)^{p} \\
& \leq 2^{\varepsilon p-\varepsilon}\left[\left(\int_{a}^{b} 1^{q} \mathrm{~d} r\right)^{\frac{1}{q}}\left(\int_{a}^{b}|K(c, r, x(r))-K(c, r, y(r))|^{p} \mathrm{~d} r\right)^{\frac{1}{p}}\right]^{p} \\
& \leq 2^{\varepsilon p-\varepsilon}(b-a)^{\frac{p}{q}}\left(\int_{a}^{b} \xi(c, r)^{p}|x(r)-y(r)|^{p} \mathrm{~d} r\right) \\
& \leq 2^{\varepsilon p-\varepsilon}(b-a)^{\frac{p}{q}}\left(\int_{a}^{b} \xi(c, r)^{p} d(x, y) \mathrm{d} r\right) \\
& \leq 2^{\varepsilon p-\varepsilon}(b-a)^{p-1} M_{s}(x, y)\left(\int_{a}^{b} \xi(c, r)^{p} \mathrm{~d} r\right) \\
& \leq M_{s}(x, y) .
\end{aligned}
$$


This implies that

$$
s^{\varepsilon} d(f x, f y) \leq M_{s}(x, y) \Rightarrow \psi\left(s^{\varepsilon} d(f x, f y)\right) \leq \psi\left(M_{s}(x, y)\right)+L \psi(N(x, y)) .
$$

Hence, $f \in \Xi_{s}(X, \alpha, \psi)$. Then all the conditions of Theorem 2.3 are satisfied and so $f$ has a fixed point in $X$. This implies that the integral equation (3.1) has a solution.

Remark 3.2. Compared with Theorem 3.1 of [19], our Theorem 3.1 has many advantages. First of all, our condition (ii) provides a natural and intrinsic characterization of simpler hypothesis than $\left(\boldsymbol{Q}_{2}\right)$ from Theorem 3.1 of [19]. As a matter of fact, our condition (3.2) is much weaker than the counterpart of Theorem 3.1 of [19]. Also, we delete the intricate function $\Gamma(t)$. Moreover, our function $\xi(c, r)$ has valid superiority since the constant $\varepsilon$ is arbitrary. Otherwise, even if $\varepsilon=3$, our $\xi(c, r)$ is also weak and straightforward.

Remark 3.3. Simple calculations show that Theorem 3.1 of [19] makes some mistakes. For instance, the following equality from the proof of this theorem is incorrect:

$$
\left(s^{3} d(f x, f y)\right)^{p}=\left(2^{3 p-3} \sup _{c \in[a, b]}|(f x)(c)-(f y)(c)|\right)^{p} .
$$

Virtually, the correct form is stated as follows:

$$
\left(s^{3} d(f x, f y)\right)^{p}=\left(2^{3 p-3} \sup _{c \in[a, b]}|(f x)(c)-(f y)(c)|^{p}\right)^{p} .
$$

Similar modifications in Corollaries 3.2 and 3.3 from [19] should be done as well.

\section{Acknowledgment}

The research was partially supported by the National Natural Science Foundation of China (11271045).

\section{References}

[1] A. Aghajani, M. Abbas, J. R. Roshan, Common fixed point of generalized weak contractive mappings in partially ordered b-metric spaces, Math. Slovaca, 64 (2014), 941-960. 1, 1.15

[2] I. A. Bakhtin, The contraction mapping principle in almost metric space, (Russian) Functional analysis, Ulyanovsk. Gos. Ped. Inst., Ulyanovsk, 30 (1989), 26-37. 1 . 1.7

[3] S. Banach, Sur les opérations dans les ensembles abstraits et leurs applications aux équations intégrales, Fund. Math., 3 (1922), 133-181. 1

[4] S. Chandok, Some fixed point theorems for $(\alpha, \beta)$-admissible Geraghty type contractive mappings and related results, Math. Sci. (Springer), 9 (2015), 127-135. 1, 1.19

[5] S. Czerwik, Contraction mappings in b-metric spaces, Acta Math. Inform. Univ. Ostraviensis, 1 (1993), 5-11. 1 1.7

[6] H.-P. Huang, S. Radenović, J. Vujaković, On some recent coincidence and immediate consequences in partially ordered b-metric spaces, Fixed Point Theory Appl., 2015 (2015), 18 pages. 1.81

[7] J. Jachymski, The contraction principle for mappings on a metric space with a graph, Proc. Amer. Math. Soc., 136 (2008), 1359-1373. 1

[8] M. Jovanović, Z. Kadelburg, S. Radenović, Common fixed point results in metric-type spaces, Fixed Point Theory Appl., 2010 (2010), 15 pages. $1.9,2$

[9] W. A. Kirk, P. S. Srinivasan, P. Veeramani, Fixed points for mappings satisfying cyclical contractive conditions, Fixed Point Theory, 4 (2003), 79-89. 1

[10] J. J. Nieto, R. Rodíguez-López, Existence and uniqueness of fixed point in partially ordered sets and applications to ordinary differential equations, Acta Math. Sin. (Engl. Ser.), 23 (2007), 2205-2212. 3

[11] R. Pant, R. Panicker, Geraghty and Ćirić type fixed point theorems in b-metric spaces, J. Nonlinear Sci. Appl., 9 (2016), 5741-5755. 1, 1.17, 1.18, $1.19,2.12$

[12] A. C. M. Ran, M. C. B. Reurings, A fixed point theorem in partially ordered sets and some applications to matrix equations, Proc. Amer. Math. Soc., 132 (2004), 1435-1443. 1 
[13] J. R. Roshan, V. Parvaneh, S. Sedghi, N. Shobkolaei, W. Shatanawi, Common fixed points of almost generalized $(\psi, \varphi)_{s}$-contractive mappings in ordered b-metric spaces, Fixed Point Theory Appl., 2013 (2013), 23 pages. 1.2

[14] P. Salimi, N. Hussain, S. Shukla, Sh. Fathollahi, S. Radenović, Fixed point results for cyclic $\alpha-\psi \varphi$-contractions with application to integral equations, J. Comput. Appl. Math., 290 (2015), 445-458. 1 ] 1.4

[15] B. Samet, C. Vetro, P. Vetro, Fixed point theorems for $\alpha \psi$-contractive type mappings, Nonlinear Anal., 75 (2012), 2154-2165. 1, 1.1, 1.3, 1.4

[16] S. Sedghi, N. Shobkolaei, J. R. Roshan, W. Shatanawi, Coupled fixed point theorems in $G_{b}$-metric spaces, Mat. Vesnik, 66 (2014), 190-201. 1.2

[17] W. Shatanawi, Fixed and common fixed point for mapping satisfying some nonlinear contraction in b-metric spaces, J. Math. Anal., 7 (2016), 1-12. 1

[18] W. Shatanawi, A. Pitea, R. Lazović, Contraction conditions using comparison functions on b-metric spaces, Fixed Point Theory Appl., 2014 (2014), 11 pages.

[19] W. Sintunavarat, Fixed point results in b-metric spaces approach to the existence of a solution for nonlinear integral equations, Rev. R. Acad. Cienc. Exactas Fís. Nat. Ser. A Math. RACSAM, 110 (2016), 585-600. 1, 1.10 $1.11,1.12,1.13,1.14,3,3.2,3.3,3$

[20] W. Sintunavarat, Nonlinear integral equations with new admissibility types in b-metric spaces, J. Fixed Point Theory Appl., 18 (2016), 397-416. 1, 1.5, 1 , 